\title{
Prostatitis crónica: Aporte del contraste endovenoso en fase tardía para su caracterización en resonancia magnética multiparamétrica de próstata
}

\section{Chronic Prostatitis: Contrast Contribution in Late Phase for its Characterization in Multiparametric-Magnetic Resonance Imaging of the Prostate}

\author{
Martina Aineseder ${ }^{1}$ Roy López Grove ${ }^{1}$ (1) Marcelo Andrés Pietrani $^{1} \quad$ Jorge Alberto Ocantos ${ }^{1}$
${ }^{1}$ Servicio de Diagnóstico por Imágenes, Hospital Italiano de Buenos Aires, Ciudad Autónoma de Buenos Aires, Argentina
Address for correspondence Roy López Grove, MD, Tte. Gral. Juan Domingo Perón, 4190, Ciudad Autónoma de Buenos Aires, Argentina Rev Argent Radiol 2020;84:115-122. (e-mail: roy.lopez@hospitalitaliano.org.ar).

\section{Resumen}

Palabras clave

- prostatitis crónica

- resonancia magnética multiparamétrica

- biopsia

- histología

- imagen por resonancia magnética

\section{Abstract}

Objetivo El objetivo de este estudio es demostrar la utilidad de una secuencia tardía post-contraste en la resonancia magnética multiparamétrica de próstata (RMMP) para caracterizar lesiones PI-RADS II.

Materiales y métodos Se analizaron en forma retrospectiva las RMMP realizadas entre enero de 2015 y diciembre de 2016. El protocolo de la RMMP fue basado en las recomendaciones del PI-RADS versión 2, y se agregó una adquisición tardía luego del dinámico post-contraste. Los reportes fueron revisados bajo la versión 2.1.

Resultados Se seleccionaron 31 pacientes que presentaron lesiones categorizadas como PIRADS II en la zona periférica, los cuales se encontraban en seguimiento del antígeno prostático específico y presentaron confirmación histológica de prostatitis crónica. Se evidenció un realce tardío de la lesión en todos los pacientes. Según los resultados histopatológicos, 30 presentaban prostatitis crónica y el restante tejido benigno (tejido fibromuscular).

Discusión La prostatitis crónica no muestra realce temprano, y presenta realce tardío debido al tejido conectivo fibroso que la compone. En la RMMP, la prostatitis puede imitar el cáncer de próstata. Agregar una adquisición tardía solo adiciona 150 segundos al estudio y podría ayudar a resolver aquellos casos inciertos categorizados como PIRADS III empleando las secuencias convencionales, debido a que el realce tardío de la lesión es altamente sugestivo de un proceso inflamatorio (PI-RADS II).

Conclusión La presencia de realce tardío es una herramienta útil para realizar un adecuado diagnóstico de una lesión PI-RADS II en la zona periférica, pudiendo evitar una biopsia innecesaria.

Objective The aim of this study is to demonstrate the utility of a post-contrast late sequence in multiparametric magnetic resonance imaging (RMMP) to characterize PIRADS II lesions. received

September 3, 2019

accepted

April 15, 2020
DOI https://doi.org/

$10.1055 / \mathrm{s}-0040-1713088$

ISSN 1852-9992.
Copyright $\odot$ 2020, Sociedad Argentina de Radiología. Publicado por Thieme Revinter Publicações Ltda., Rio de Janeiro, Brazil. Todos los derechos reservados.
License terms

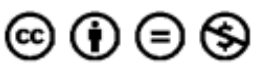




\author{
Keywords \\ - chronic Prostatitis \\ - multiparametric- \\ magnetic \\ resonance \\ imaging \\ - biopsy \\ - histology \\ - magnetic \\ resonance \\ imaging
}

Materials and methods The RMMPs performed between January 2015 and December 2016 were retrospectively analyzed. The RMMP protocol was based on the recommendations of the PI-RADS version 2 , and a late acquisition was added, after the dynamic post-contrast. The reports were reviewed under the version 2.1.

Results 31 patients with PI-RADS II lesions in the peripheral zone were selected, who were in prostate specific antigen follow-up and had histological confirmation of chronic prostatitis. A late enhancement of the lesion was evidenced in all patients. According to the histopathological results, 30 had chronic prostatitis and the remaining benign tissue (fibromuscular tissue).

Discussion Chronic prostatitis does not show early enhancement, and presents late enhancement due to its fibrous connective tissue. In RMMP, prostatitis may mimic prostate cancer. Adding a late sequence only adds 150 seconds to the study and could help to resolve those uncertain cases categorized as PI-RADS III using traditional sequences because the late enhancement of the lesion is highly suggestive of an inflammatory process (PI-RADS II).

Conclusion The presence of late enhancement is a useful tool to perform an adequate diagnosis of a PI-RADS II lesion in the peripheral zone, helping to avoid an unnecessary biopsy.

\section{Introducción}

La resonancia magnética multiparamétrica de próstata (RMMP) ha revolucionado el estudio de la patología prostática, y el sistema de datos e informe en imagen prostática versión 2 (Prostate Imaging and Reporting and Data System, PI-RADS v2) ha producido la unificación de diferentes criterios. ${ }^{1,2}$ Sin embargo, en múltiples casos, es dificultoso arribar a un diagnóstico. ${ }^{3}$ La v2.1 recomienda varios ajustes menores destinados a simplificar la evaluación y reducir la variabilidad entre los lectores, sin cambiar el alcance o los principios generales del sistema original. ${ }^{4}$

Es ampliamente reconocido en la literatura que los hallazgos de prostatitis en RMMP pueden ser similares a los de cáncer de próstata, y la diferenciación entre esas dos entidades puede ser dificultosa.

A pesar de la actualización del PI-RADS, aún es difícil diferenciar entre ambas patologías, debido a que las dos presentan baja señal ponderadas en T2 y ADC, hiperintensidad en difusión (DWI) y realce con el contraste sin importar su localización en la zona periférica (ZP) o transicional. ${ }^{5,6}$

La prostatitis crónica es un trastorno que se caracteriza por la inflamación crónica de la próstata, que puede causar un aumento del antígeno prostático específico (PSA). Sin embargo, es dificultoso su diagnóstico porque no hay síntomas ni marcadores sanguíneos específicos. ${ }^{7,8}$ La prevalencia de la prostatitis es alta, con una tasa global del $8,2 \%{ }^{7}$ Es por eso que, ante la duda diagnóstica, en algunos pacientes puede ser indicada una biopsia transrectal guiada por ecografía o fusión RM-ecografía. ${ }^{9-11}$ Una alternativa diagnóstica que evite estudios invasivos es necesaria.

El objetivo de este estudio es demostrar la utilidad de agregar una secuencia tardía post-contraste endovenoso en la RMMP, herramienta que puede resultar de utilidad para caracterizar lesiones PI-RADS II y sin tener necesidad de emplear procedimientos invasivos, como la biopsia transrectal.

\section{Materiales y métodos}

Se analizaron en forma retrospectiva las RMMP realizadas entre enero de 2015 y diciembre de 2016. El estudio fue aprobado por el Comité de Ética de la institución, que autorizó la exención del consentimiento informado debido a las características del estudio. Se seleccionaron aquellos pacientes que presentaban lesiones PI-RADS II en la ZP con confirmación de prostatitis crónica con biopsia dirigida a la lesión.

Los criterios de inclusión fueron pacientes mayores de 45 años de edad en vigilancia con un aumento progresivo del PSA, PSA aumentado $(>2.5 \mathrm{ng} / \mathrm{ml})$ en más de una lectura consecutiva sin otros factores de riesgo asociados, un nódulo sospechoso identificado por tacto rectal y/o antecedentes familiares de cáncer de próstata.

Los pacientes con biopsia prostática previa o diagnóstico confirmado de cáncer de próstata fueron excluidos.

Todas las RMMP fueron interpretadas por un especialista en radiología genitourinaria de RMMP de 11 años de experiencia $\mathrm{y}$ los reportes de los informes fueron estandarizados en base a PI-RADS v2 y revisados con los criterios de la v2.1. Las biopsias fueron interpretadas por dos patólogos utilizando un informe estandarizado.

La preparación previa incluyó un ayuno de 6 horas. Los estudios fueron realizados utilizando un protocolo estándar específico en un resonador 1.5 Tesla (Siemens MAGNETOM Avanto ${ }^{\circ}$; Siemens Medical Solutions, Erlangen, Alemania) usando una bobina corporal de superficie con pacientes en posición supina. Se administró hioscina $10 \mathrm{mg}$ por vía endovenosa inmediatamente previo a la realización del 
estudio. Se utilizó la superficie posterior de la próstata como referencia para la orientación. Las imágenes transversales se orientaron perpendiculares a la superficie posterior de la próstata (desde la base al ápex) y las coronales paralelas al plano mencionado (desde la sínfisis al recto).

El protocolo de la RMMP fue basado en las recomendaciones del PI-RADS v2: secuencias ponderadas en T2, T1, DWI/coeficiente de difusión aparente (ADC) con valores de b50, 400, 800 y $1600 \mathrm{~s} / \mathrm{mm}^{2}$ y estudio dinámico con contraste endovenoso. Se utilizó gadolinio (gadoterato de meglumina) a una dosis de $0,1 \mathrm{mmol} / \mathrm{kg}$ con un flujo de $3 \mathrm{ml} / \mathrm{s}$ seguido de $20 \mathrm{ml}$ de solución salina a un flujo similar y se realizaron secuencias con la técnica Volume Interpolated Breath-hold Examination (flash 3D VIBE) orientada en plano axial, usando adquisiciones consecutivas cada 20 segundos, durante 2 minutos. Además, se agregó una adquisición tardía ponderada en $\mathrm{T} 1$ con saturación grasa (FS) en planos axial y sagital limitada a la próstata a partir de los 3 minutos de inyección del gadolinio, con una duración de 75 segundos por secuencia (- Tabla 1). Se empleó el mismo protocolo en todos los pacientes incluidos, independiente del motivo de estudio.

Los criterios para caracterizar la prostatitis crónica fueron lesiones en la ZP hipointensas ponderadas en T2, hiperintensas en DWI con restricción en el ADC y sin realce en secuencias tempranas con el contraste y morfología lineal, en cuña o difusa. ${ }^{4}$ Se interpretó como realce tardío positivo persistente la presencia de realce en fase tardía subjetivamente mayor que las áreas de ZP adyacentes, hallazgo coincidente con áreas de baja señal ponderada en $\mathrm{T} 2$.

\section{Resultados}

Se catalogaron 44 RMMP como PI-RADS II durante ese período. Se seleccionaron 31 pacientes entre 46 y 71 años de edad, los cuales realizaron una RMMP con contraste endovenoso y presentaban una lesión PI-RADS II en la ZP, que realizaron seguimiento del PSA en nuestra institución y tenían una biopsia que confirmaba la presencia de prostatitis crónica. Se excluyeron los restantes por presentar lesiones en la zona transicional. Comparando el análisis con la v2.1, no se encontraron diferencias en la categorización del PI-RADS.

El volumen promedio de la glándula prostática calculado por elipsoide en RMMP fue de $79 \mathrm{cc}$ (en un rango de 70 a 200 cc), con un PSA de $7 \mathrm{ng} / \mathrm{ml}$ (en un rango de 2,6 a $16,7 \mathrm{ng} / \mathrm{ml}$ ). Ninguno de los pacientes presentaba historia de cáncer de próstata ni infecciones activas.

Una biopsia transrectal guiada por ecografía fue realizada en todos los pacientes luego de la realización de la RMMP en un promedio de tiempo de 3,1 semanas (rango de 1,2 a 6,6 semanas). En relación a las biopsias, 25 fueron realizadas mediante fusión con software y 6 mediante fusión cognitiva. Según los resultados histopatológicos, 30 presentaban prostatitis crónica y un paciente presentó tejido benigno, el cual fue informado como tejido fibromuscular.

En la RMMP, la presencia de realce tardío de la lesión se encontró en todos los pacientes ( $\mathbf{- F i g}$. 1). Ese patrón es consistente con prostatitis crónica, traduciendo la presencia de signos de inflamación crónica (-Fig. 2).

\section{Discusión}

Las lesiones de la prostatitis crónica en RMMP se presentan como áreas difusas en forma unilateral o bilateral, con poco o nulo efecto de masa o con forma de cuña o lineal con baja intensidad de señal ponderada en T2 en la zona periférica. ${ }^{1-4}$ Debido a la destrucción de la glándula prostática, que genera un descenso en el gradiente de difusión del agua, se pueden observar valores bajos de ADC. ${ }^{4}$ Aunque en la prostatitis crónica la disminución de la señal en el ADC no suele ser tan pronunciada ni tan focal como en el cáncer, su utilidad es limitada debido a que existe superposición de los valores de ADC entre ambas patologías. ${ }^{3}$

El realce dinámico con contraste se basa en la captación y liberación del gadolinio por los tejidos, y ayuda en la

Tabla 1 Protocolo de RM multiparamétrica de próstata con contraste endovenoso y evaluación de la utilidad de cada secuencia

\begin{tabular}{|l|l|l|l|l|l|}
\hline Secuencia & Plano & Región & $\begin{array}{l}\text { Campo de } \\
\text { visión }(\mathbf{m m})\end{array}$ & $\begin{array}{l}\text { Espesor de } \\
\text { corte }(\mathbf{m m})\end{array}$ & Utilidad \\
\hline T2 & Axial & Pelvis & 220 & 4 & $\begin{array}{l}\text { Nódulos linfáticos retroperitoneales } \\
\text { y pélvicos }\end{array}$ \\
\hline T1 & Axial & Próstata & 160 & 3 & Hemorragia. Delimita la glándula \\
\hline T2 & $\begin{array}{l}\text { Axial, coronal } \\
\text { y sagital }\end{array}$ & Próstata & 180 & 3 , sin gap & Discrimina zonas anatómicas prostáticas \\
\hline DWI/ADC* & Axial & Pelvis & 220 & 4 , sin gap & Densidad celular del tejido \\
\hline $\begin{array}{l}\text { T1 FS 3D dinámico } \\
\text { con contraste } \\
\text { (perfusión)** }\end{array}$ & Axial & Próstata & 250 & 3 , sin gap & Realce \\
\hline $\begin{array}{l}\text { T1 FS con contraste. } \\
\text { T1 TSE*** }\end{array}$ & $\begin{array}{l}\text { Axial } \\
\text { y sagital }\end{array}$ & Próstata & 160 & 3 & $\begin{array}{l}\text { Isquemia post-embolización. } \\
\text { Realce tardío PI-RADS II**** }\end{array}$ \\
\hline
\end{tabular}

*Valores de b: 50, 400, 800, $1600 \mathrm{~s} / \mathrm{mm}^{2}$. DWl: difusión ADC: coeficiente aparente de difusión.

**Adquisiciones consecutivas, cada 20 segundos por 2 minutos. FS: saturación grasa.

***Planos iguales a secuencias ponderadas en T2 de la próstata. TSE: turbo spin echo.

${ }^{* * * *}$ Secuencia realizada en nuestra institución. 

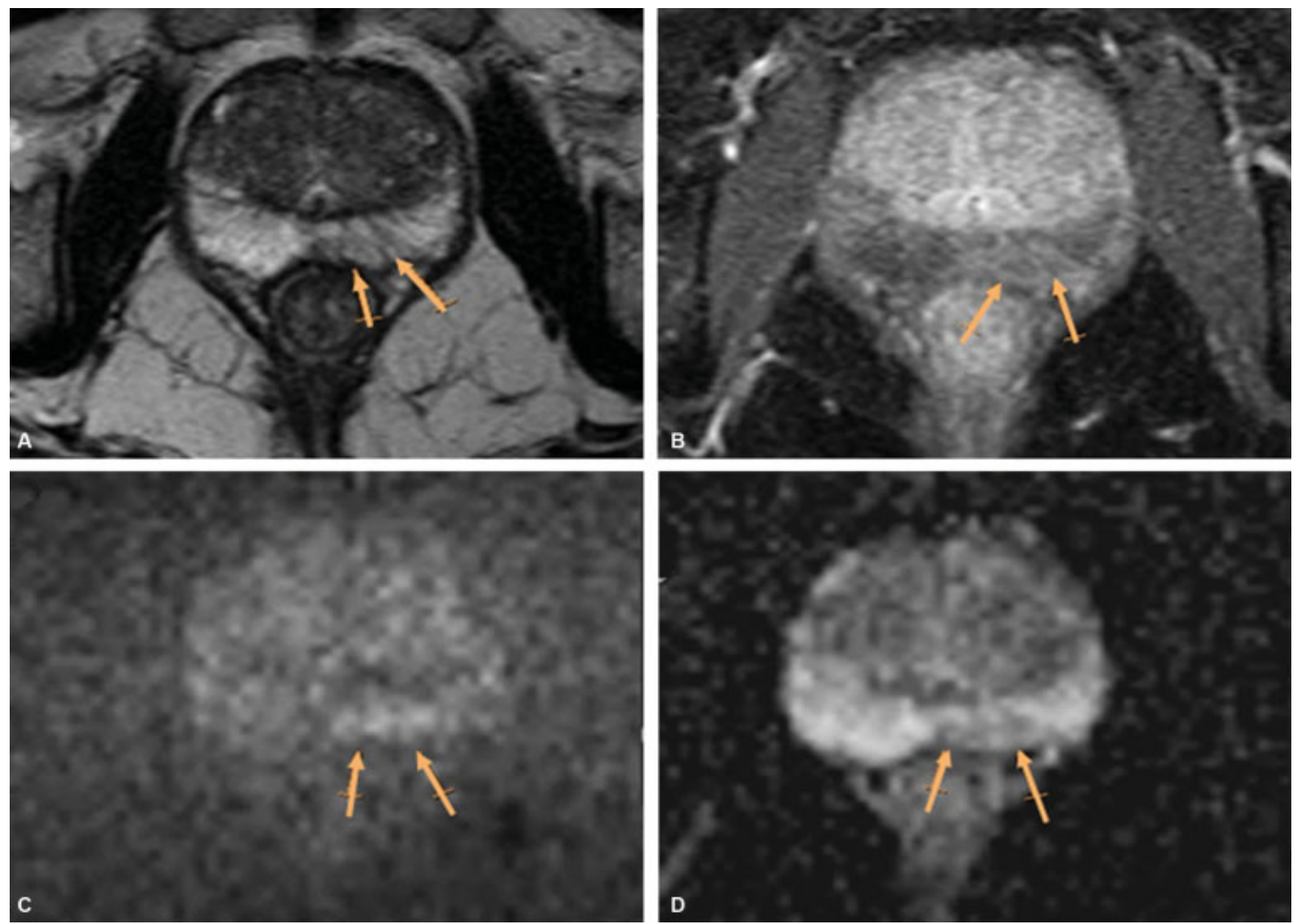

Fig. 1 Paciente de 66 años en estudio por aumento del PSA, antecedente de hiperplasia prostática benigna. Lesión en forma de cuña en región posteromedial izquierda de la ZP (flechas), hipointensa ponderada en T2 (A), con realce tardío en secuencia ponderada en T1 FS tardía con contraste (B). Presenta una restricción leve en DWI con altos valores de b, sin representación significativa en ADC (C, D). Fue categorizada como PI RADS II. ZP: Zona periférica. FS: Saturación grasa. DWI: Difusión. ADC: Coeficiente de difusión aparente.
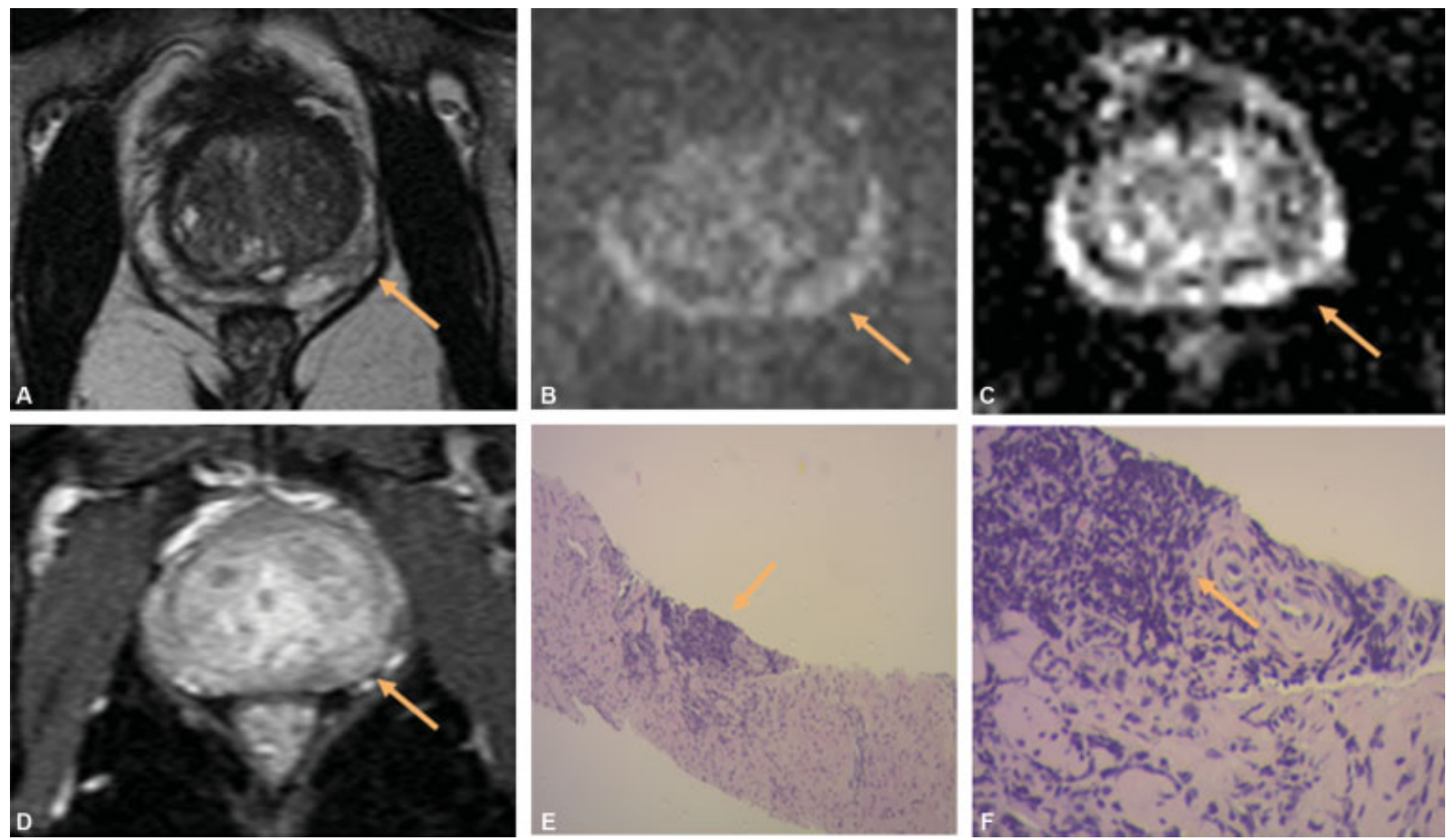

Fig. 2 Paciente de 71 años en estudio por PSA de 2,46 ng/mL. Lesión semilunar PI RADS II en la ZP posterolateral izquierda (flecha), hipointensa ponderada en T2 (A). No se observa ninguna lesión focal en el DWI 800 ni mapa de ADC (B, C). Realce en secuencia tardía (D). Se realizó biopsia prostática en la que se evidenciaron numerosos linfocitos en el estroma entre las glándulas (10x E, 40x F). DWI: Difusión. ADC: coeficiente de difusión aparente. 
diferenciación de los tejidos en base a su evaluación funcional. En la prostatitis crónica, existe proliferación de vasos sanguíneos a lo largo del tejido conectivo, resultando en aumento del suministro sanguíneo. Sin embargo, la arquitectura de la microcirculación se mantiene en forma relativa. ${ }^{12}$ La prostatitis crónica usualmente no muestra realce temprano, y presenta realce tardío debido al tejido conectivo fibroso ( - Fig. 3). ${ }^{5,13}$ Sin embargo, también puede existir captación temprana durante el estudio dinámico con el contraste resultando a un falso positivo para cáncer, aunque no es tan marcado. ${ }^{14}$

En las lesiones prostáticas, es indispensable distinguir entre benignas y malignas, no solo por el pronóstico sino también para evitar procedimientos invasivos. El hallazgo constante de realce tardío en lesiones PI-RADS II, así como la ausencia de realce temprano, puede ser utilizado como una herramienta para el radiólogo, para arribar a un adecuado diagnóstico y también para evitar biopsias innecesarias.

El PI-RADS v2 de 2015 y su revisión en 2019 (v2.1) ha sido el estándar para categorizar el riesgo de cáncer de próstata por imágenes. Sin embargo, la prostatitis puede imitar el cáncer de próstata en RMMP, especialmente en pacientes con prostatitis crónica. ${ }^{7}$ Aunque el realce tardío no se utiliza normalmente en los protocolos de RMMP para la detección de patología prostática, es una de las mejores secuencias que muestra cambios antes y después de la embolización en casos de hiperplasia prostática benigna. ${ }^{7}$ Es importante resaltar que en nuestra institución se realiza el mismo protocolo tanto para pacientes con sospecha de cáncer de próstata como en aquellos estudiados por hiperplasia prostática benigna o posterior a la embolización. ${ }^{15}$ En esos últimos, es importante contar una secuencia de adquisición tardía para evaluar infartos prostáticos en controles postembolización y valorar la respuesta al tratamiento. ${ }^{16,17}$

Dado el rol secundario otorgado al gadolinio que la experiencia ha demostrado en la categorización general del PI-RADS, hay un interés creciente en realizar RM de próstata usando solamente T2 y DWI, denominado RM biparamétrica. $^{4}$ Sin embargo, solo puede ser útil en estudios de alta calidad y libres de artefactos, lo cual no siempre ocurre, en particular cuando no se emplea una bobina endorrectal. ${ }^{18}$ Por lo tanto, agregar una secuencia post-contraste tardía puede ayudar en casos de pacientes con
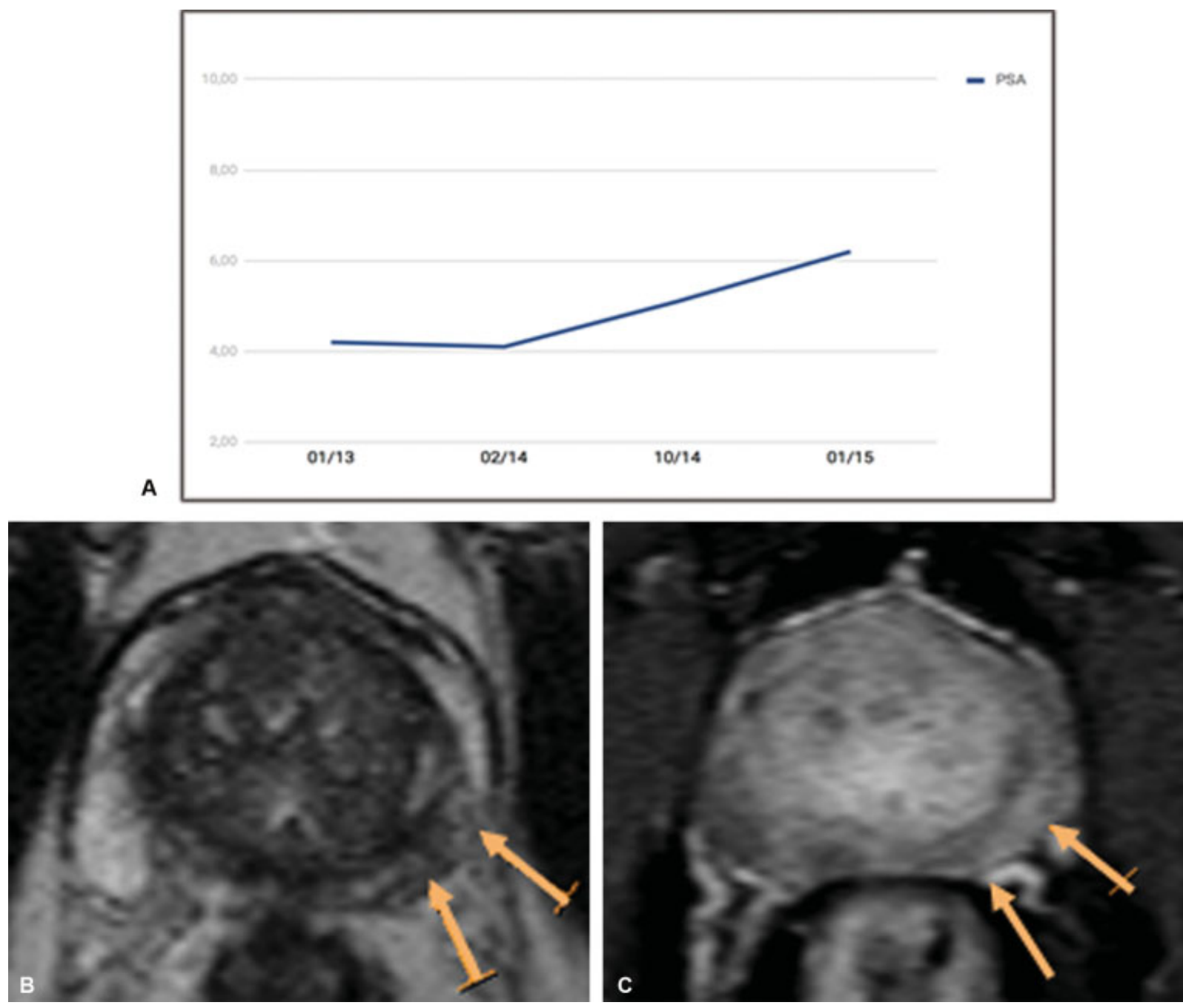

Fig. 3 Paciente de 60 años de edad en seguimiento por aumento del PSA. Curva de aumento de PSA (A). Lesión lineal PI RADS II en la ZP posterolateral izquierda, hipointensa ponderada en T2 (flechas) (B). Realce tardío lineal ponderado en T1 FS tardío (C). ZP: Zona periférica. 
reemplazo de cadera, artefacto significativo de DWI, o al análisis por especialistas menos experimentados.

En la v2.1 del PI-RADS hay especificaciones de modificaciones en la interpretación para las zonas transicional, zona central y estroma fibromuscular, pero no tan riguroso para la $\mathrm{ZP}^{4}{ }^{4}$ En esa última versión, se ha modificado la definición de la puntuación 2 y 3 para DWI con respecto a la v2. La categoría 2 de DWI se define ahora como un área lineal en forma de cuña que es hiperintensa en DWI de alto valor b y/o hipointensa en ADC. ${ }^{4}$ Ella es similar a la puntuación del T2 para la categoría 2 de PZ y debe ayudar a reducir la puntuación potencial de la inflamación como resultado 3 en DWI, que a su vez puede ser elevado a una categoría PI-RADS IV si demuestra realce focal temprano asociado. ${ }^{19}$ La puntuación 3 se define como un área focal de señal aumentada en alto valor b de DWI y/o baja señal en ADC, pudiendo ser marcadamente hipointensa en ADC o hiperintensa en alto valor b de DWI pero no en ambas. Las puntuaciones 1,4 y 5 no se han modificado. Al analizar nuestros pacientes con la v2.1 no encontramos diferencias en la categorización del PI-RADS final.

Realizar una secuencia tardía que demuestre el realce tardío es útil en aquellos casos en los cuales no es posible categorizar con precisión una lesión PI-RADS II con las secuencias convencionales, o cuando la difusión no se puede realizar o es insuficiente. Agregar una secuencia tardía solo adiciona 150 segundos al estudio y permitiría evaluar aquellos casos inciertos categorizados como PI-RADS III, evitando así una posible biopsia sobre una lesión PI-RADS II en ZP (-Fig. 4). . $^{8}$

Es importante recordar que ese hallazgo no se encuentra avalado por el PI-RADS v2 ni v2.1 y tampoco modifica la categorización. Sin embargo, podría ser planteado como un comentario o una sugerencia en el informe médico, o bien plantear la discusión cuando se debate la probable terapéutica del paciente.

En nuestro grupo de pacientes, la secuencia tardía fue realizada en todos los casos y, junto con la información aportada por las demás secuencias, contribuyó a asegurar una adecuada categorización (-Fig. 5).

Como limitaciones de nuestro estudio, debe notarse la falta de utilización de una bobina endorrectal que, combinada con la bobina de superficie, permite obtener estudios de alta resolución de la pelvis. ${ }^{2}$ Sin embargo, es importante recordar que su uso no es obligatorio debido a que puede aumentar el costo y el tiempo del examen, deformar la glándula, introducir artefactos, además de ser incómodo para los pacientes y aumentar su reticencia a someterse a la RMMP. Otra limitación posible es que no se utilizó un resonador 3T. La adquisición de imágenes de $3.0 \mathrm{~T}$ proporciona una mejor relación señal/ruido con una adquisición más rápida de imágenes de alta calidad, con o sin el uso de una bobina endorrectal. ${ }^{20}$ Otro potencial limitante es la adquisición de las imágenes con el protocolo recomendado en la v2 debido a la
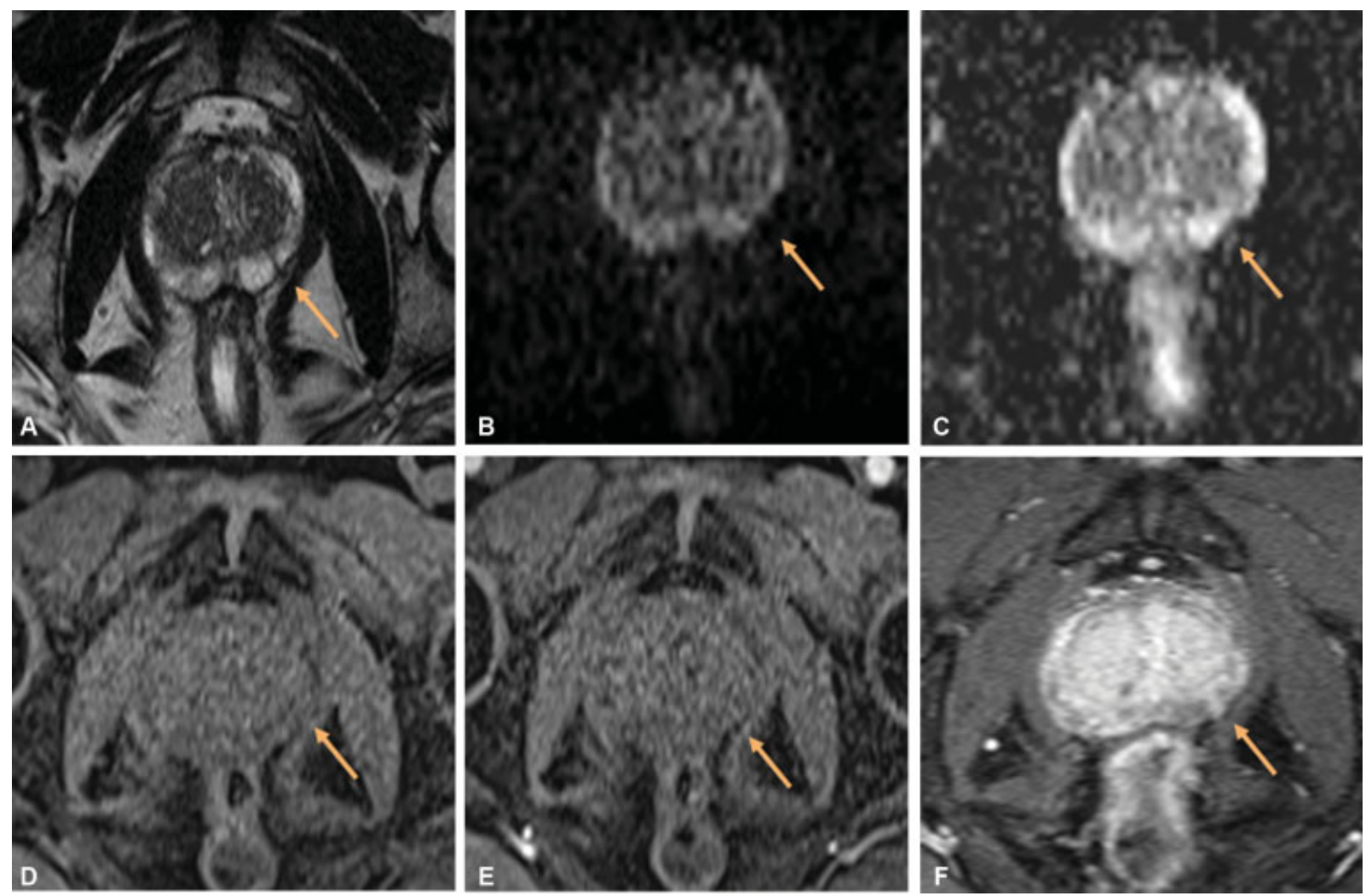

Fig. 4 Paciente de 59 años en estudio por aumento del PSA. Lesión lineal PI RADS II en la ZP posterolateral izquierda (flecha), hipointensa ponderada en T2 (A), con una restricción leve en ADC pero sin hallazgo focal en valores altos de B en DWI (B, C). No se observa anormalidad focal ponderada en T1 FS (D) y secuencias tempranas del dinámico tras la administración de contraste (E). Realce lineal tardío en secuencia tardía (F). ZP: Zona periférica. DWI: Difusión. ADC: coeficiente de difusión aparente. FS: Saturación grasa. 

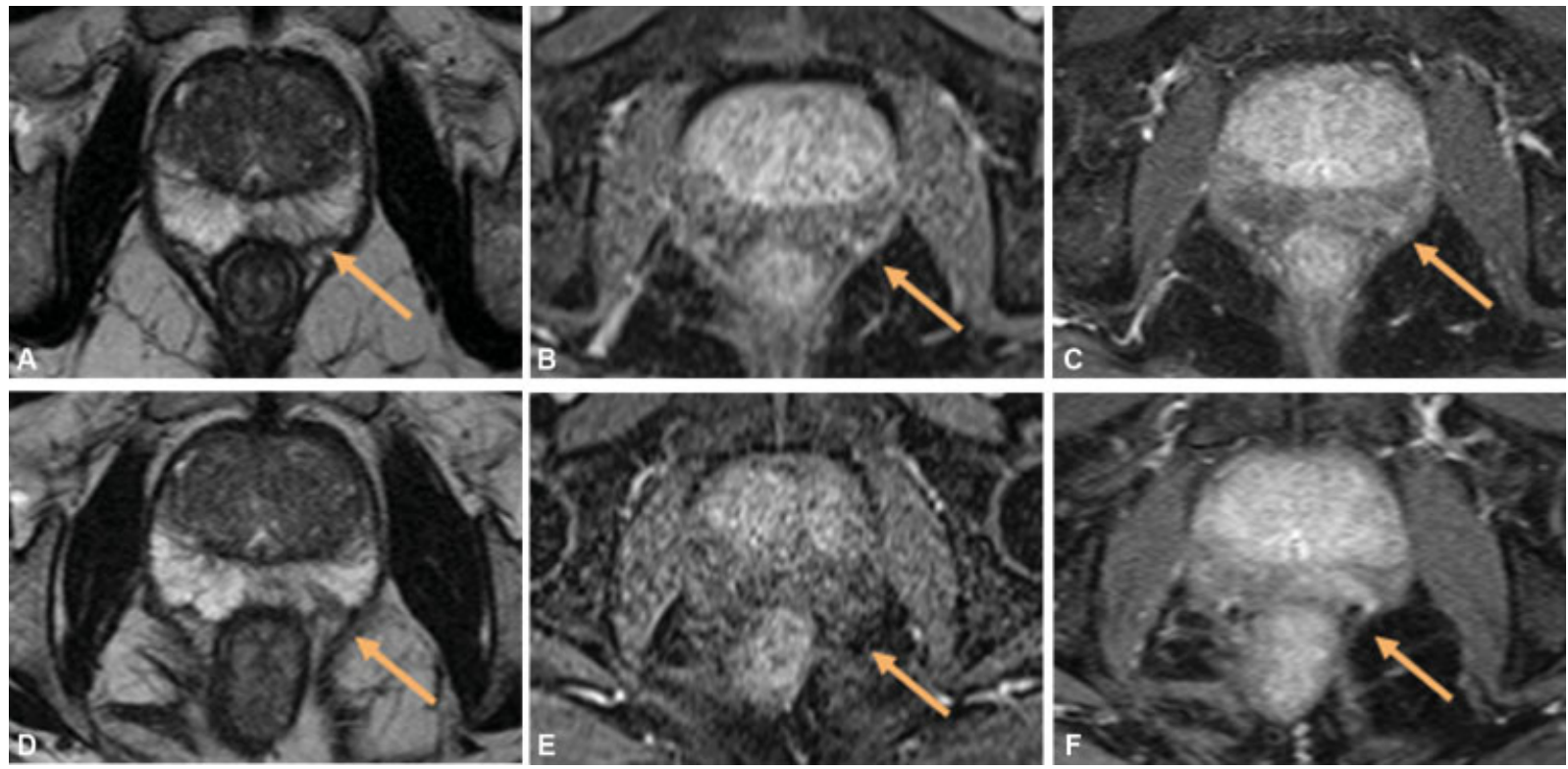

Fig. 5 Paciente en seguimiento por aumento del PSA, con valor inicial de $4 \mathrm{ng} / \mathrm{ml}$ y de 5,2 $\mathrm{ng} / \mathrm{ml}$ al año. Lesión lineal PI RADS II en la ZP posteromedial izquierda (flecha), hipointensa ponderada en T2 (A). No se observan lesiones focales en las secuencias dinámicas tempranas tras la administración de contraste (B). Realce lineal tardío en secuencia tardía (C). En el estudio del año siguiente, se observa mayor hipointensidad ponderada en T2 (D), persistiendo sin realce temprano (E) y con mayor realce tardío (F). ZP: Zona periférica. FS: Saturación grasa.

fecha de realización de las RMMP. Sin embargo, el análisis de los estudios y en particular de la secuencia tardía postcontraste no modifica significativamente su interpretación. Por último, el análisis fue realizado por un solo médico especialista de forma subjetiva. Sería útil un análisis independiente por dos observadores y la valoración interobservador.

\section{Conclusión}

La RMMP puede demostrar características de la prostatitis crónica. El hallazgo de un realce tardío con el contraste constituye un signo indicador de prostatitis crónica y es una herramienta complementaria útil para diagnosticar en forma adecuada una lesión PI-RADS II en la ZP.

\section{Responsabilidades éticas}

Este es un trabajo observacional retrospectivo el cual cumplió los principios éticos de la Declaración de Helsinki en su última revisión de octubre del año 2000.

Protección de personas y animales. Los autores declaran que para esta investigación no se han realizado experimentos en seres humanos ni en animales.

Confidencialidad de los datos. Los autores declaran que han seguido los protocolos de su centro de trabajo sobre la publicación de datos de pacientes.

\section{Derecho a la privacidad y consentimiento informado.} Los autores declaran que en este artículo no aparecen datos de pacientes.

\section{Conflicto de Intereses}

Los autores declaran no tener ningún conflicto de intereses.

\section{Bibliografía}

1 Weinreb JC, Barentsz JO, Choyke PL, Cornud F, Haider MA, Macura $\mathrm{KJ}$, et al. PI-RADS prostate imaging - reporting and data system: 2015, Version 2. Eur Urol. 2016;69(01):16-40

2 Vilanova JC, Comet J, Garcia-Figueiras R, Barceló J, Boada M. Utilidad de la resonancia magnética en el cáncer de próstata. Radiologia (Madr) 2010;52(06):513-524

3 Nagel KN, Schouten MG, Hambrock T, Litjens GJ, Hoeks CM, ten Haken B, et al. Differentiation of prostatitis and prostate cancer by using diffusion-weighted MR imaging and MR-guided biopsy at 3 T. Radiology. 2013;267(01):164-172

4 Turkbey B, Rosenkrantz AB, Haider MA, Padhani AR, Villeirs G, Macura KJ, et al. Prostate Imaging Reporting and Data System Version 2.1: 2019 Update of Prostate Imaging Reporting and Data System Version 2. Eur Urol. 2019;76(03):340-351

5 Meier-Schroers M, Kukuk G, Wolter K, Decker G, Fischer S, Marx C, et al. Differentiation of prostatitis and prostate cancer using the Prostate Imaging-Reporting and Data System (PI-RADS). Eur J Radiol. 2016;85(07):1304-1311

6 Jyoti R, Jina NH, Haxhimolla HZ. In-gantry MRI guided prostate biopsy diagnosis of prostatitis and its relationship with PIRADS V.2 based score. J Med Imaging Radiat Oncol. 2017;61(02): 212-215

7 Krieger JN, Nyberg L Jr, Nickel JC. NIH consensus definition and classification of prostatitis. JAMA. 1999;282(03):236-237

8 Nickel JC. Clinical evaluation of the man with chronic prostatitis/ chronic pelvic pain syndrome. Urology. 2002;60(6, Suppl)20-22, discussion 22-23

9 Franiel T, Stephan C, Erbersdobler A, Dietz E, Maxeiner A, Hell N, et al. Areas suspicious for prostate cancer: MR-guided biopsy in patients with at least one transrectal US-guided biopsy with a negative finding-multiparametric MR imaging for detection and biopsy planning. Radiology. 2011;259(01):162-172

10 Galosi AB, Tiroli M, Cantoro D, Conti A, Muzzonigro G. Biopsy of the anterior prostate gland: technique with end-fire transrectal ultrasound. Arch Ital Urol Androl. 2010;82(04):248-252

11 Cornelis F, Rigou G, Le Bras Y, Coutouly X, Hubrecht R, Yacoub M, et al. Real-time contrast-enhanced transrectal US-guided prostate biopsy: diagnostic accuracy in men with previously negative 
biopsy results and positive MR imaging findings. Radiology. 2013; 269(01):159-166

12 Lovett K, Rifkin MD, McCue PA, Choi H. MR imaging characteristics of noncancerous lesions of the prostate. J Magn Reson Imaging. 1992;2(01):35-39

13 Krieger JN, McGonagle LA. Diagnostic considerations and interpretation of microbiological findings for evaluation of chronic prostatitis. J Clin Microbiol. 1989;27(10):2240-2244

14 Lovegrove CE, Matanhelia M, Randeva J, Eldred-Evans D, Tam H, Miah $S$, et al. Prostate imaging features that indicate benign or malignant pathology on biopsy. Transl Androl Urol. 2018;7(Suppl 4):S420-S435

15 Borensztein M, Adri D, Tobía I, Bergero M, Martínez P, Ocantos J. Biopsia de próstata transrrectal bajo guía ecográfica y fusión con resonancia magnética: experiencia inicial en pacientes con biopsia previa negativa. Rev Argent Urol. 2018;83(03):102-108
16 Vargas HA, Wassberg C, Akin O, Hricak H. MR imaging of treated prostate cancer. Radiology. 2012;262(01):26-42

17 Frenk NE, Baroni RH, Carnevale FC, Gonçalves OM, Antunes AA, Srougi M, et al. MRI findings after prostatic artery embolization for treatment of benign hyperplasia. AJR Am J Roentgenol. 2014; 203(04):813-821

18 Caglic I, Hansen NL, Slough RA, Patterson AJ, Barrett T. Evaluating the effect of rectal distension on prostate multiparametric MRI image quality. Eur J Radiol. 2017;90:174-180

19 Barrett T, Rajesh A, Rosenkrantz AB, Choyke PL, Turkbey B. PIRADS version 2.1: one small step for prostate MRI. Clin Radiol. 2019;74(11):841-852

20 Barentsz JO, Richenberg J, Clements R, Choyke P, Verma S, Villeirs $\mathrm{G}$, et al; European Society of Urogenital Radiology. ESUR prostate MR guidelines 2012. Eur Radiol. 2012;22(04):746-757 